\title{
Corrigendum
}

\section{First feed affects the expressions of microRNA and their targets in Atlantic cod - CORRIGENDUM}

\author{
Teshome Tilahun Bizuayehu, Tomasz Furmanek, Ørjan Karlsen, Terje van der Meeren, \\ Rolf Brudvik Edvardsen, Ivar Rønnestad, Kristin Hamre, Steinar D. Johansen and Igor Babiak
}

(First published online 21 March 2016)

doi:10.1017/S0007114516000155, Published by Cambridge University Press, February 2016

The information in the last paragraph on page 7 regarding DUSP5 is incorrect, and the citation (55) is incorrect. We apologise for this error that arose because of ambiguity in naming of the gene of interest.

The sentence:

'Among them, dusp5 is a negative regulator of p38 and stress-activated protein kinases/Jun amino-terminal kinases (SAPK/JNK) (55), which are involved in signal transduction pathways with diverse biological functions (56).

Should be replaced with sentence:

'Among them, dusp5 is a mitogen- and stress-inducible nuclear phosphatase that specifically interacts and inactivates ERK2 (55), which is activated by growth factors and phorbol esters (56).'

Also, the citation 55 should be replaced with the citation:

55. Mandl M, Slack DN \& Keyse SM (2005) Specific inactivation and nuclear anchoring of extracellular signal-regulated kinase 2 by the inducible dual-specificity protein phosphatase DUSP5. Mol Cell Biol 25, 1830-1845.

\section{Reference}

1. Bizuayehu TT, Furmanek T, Karlsen $\varnothing$, et al. (2016) First feed affects the expressions of microRNA and their targets in Atlantic cod. Br J Nutr 115, 1145-1154. available on CJO2016. doi:10.1017/S0007114516000155. 